\title{
«FILOSOFAR»: ENTORNO VIRTUAL PARA EL APRENDIZAJE EN EL FILOSOFAR
}

\author{
Germán Vargas Guillén \\ Sonia Cristina Gamboa S. \\ Universidad Pedagógica Nacional
}

\section{Resumen}

Nuestra sociedad actual, tecnológicamente sedimentada, requiere apropiar procesos de aprendizaje virtual en programas de pregrado y posgrado que preparen a sus egresados para un desempeño adecuado en ésta. La institucionalización de la investigación y la enseñanza de la filosofía en la Universidad Pedagógica Nacional asumió los ambientes virtuales de aprendizaje como pieza fundamental en el diseño de sus programas. «'Filosofar': Entorno virtual para el aprendizaje en el filosofar» presenta una propuesta para llevar a cabo este fin. Es así como en ésta se caracteriza tanto el enfoque teórico del proyecto como las estrategias de aprendizaje y los entornos que se han desarrollado dentro del mismo. Finalmente, explicita cómo se lleva a cabo tanto el proceso de construcción de conocimientos, como la validación del mismo.

\section{Palabras clave}

Filosofía, aprendizaje de la filosofía, estrategias de aprendizaje, aprendizaje virtual.

\section{Abstract}

Our present society, tecnologically settle, requires to adapt virtual learnin processes to the undergraduate and postgraduate programs to prepare the students for an appropiate performance in the society. The research institutionalizing and the philosophy teaching at the Universidad Pedagogica Nacional assumed the learning virtual atmosphere as a fundamental part in the design of their programs. "Philosophizing virtual atmosphere for learning while philosophizing" presents a proposal to carry out this purpose. Thereby, it is characterized by the theoretical focus of the project as well as the learning strategies and the atmospheres which have been developed within ifself. Finally, it explains how the process of knowledge building is carried out as well as the validation of itself. 


\section{Index terms}

Philosophy, philosophy learning, virtual learning, learning strategies

...no es posible aprender filosofía, pues

¿dónde está, quién la posee y en qué podemos reconocerla?

Sólo se puede aprender a filosofar.

I. Kant

Crítica de la razón pura, A 837

\section{Contexto de referencia}

El proyecto Institucionalización de la investigación y la enseñanza de la filosofía, que lidera actualmente la Facultad de Humanidades de la Universidad Pedagógica Nacional, ha creado un departamento para tal fin. Éste tiene a su cargo suplir las necesidades de formación filosófica como parte del enfoque pedagógico de los programas que actualmente ofrece la Universidad, así como la creación y puesta en marcha de programas de pregrado y posgrado, $\mathrm{y}$ de proyectos de investigación en el área de la filosofía y su enseñanza.

El problema de la Investigación y la enseñanza de la filosofía-que pretende lograr la formación de futuros licenciados con habilidades de orden cognitivo como el análisis, la interpretación y la crítica- se convierte, entonces, para el proyecto, en un problema de aprendizaje de la filosofía. Se genera, pues, con ello, un cambio de paradigma: de la enseñanza al aprendizaje. Es así como en el marco de proyectos de investigación en el área de la didáctica de la filosofía, que se adelantan actualmente, se evidencia la necesidad de plantear estrategias que permitan al estudiante alcanzar las habilidades mencionadas.

\section{Enfoque teórico}

Teóricamente, esta investigación da unos pasos en la dirección de la resolución de problemas aplicada al aprendizaje de la filosofía. Se adopta la posición que a este respecto fue formulada por Newell y Simon $^{1}$, y que en el campo de la representación computacional de dilemas morales se ha desarrollado en la investigación computacional $^{2}$.

Para hacer énfasis dentro de los procesos de resolución al ítem "cambios de estado", se ha recurrido a la "representación de conocimiento" ${ }^{3}$ con estrategias como "mapas con-

1 NEWELL, Allen y SIMON, Herbert A. “La ciencia de la computación”. En: Filosofía de la inteligencia artificial. México: F.C.E., 1994.

2 VARGAS GUILLÉN, Germán. La representación computacional de dilemas morales: Investigación fenomenológica de epistemología experimental. Tesis doctoral dirigida por el prof. Luis Facundo Maldonado Granados. Bogotá: Universidad Pedagógica Nacional, 2004.

3 NOVAK, J. D. y GOWIN, D. B. Learning how to learn. New York y Cambridge: Cambridge University Press, 1984; GLYMOUR, Clark. thinking Things throught: an introduction to philosophical issues and achievements. Cambrige: MIT Press, 1997. 
ceptuales”, “redes semánticas”, “lógica clausal” y “Árbol de Porfirio”4.

Igualmente, el enfoque teórico toma en consideración la doctrina de “aprendizaje colaborativo”, así como la concepción de "aprendizaje en red"5.

Al cabo, pues, la investigación parte del supuesto esencial de que el aprendizaje de la filosofía tiene que ver, más que con la erudición filosófica, con la "investigación de las cosas mismas”, con su análisis ${ }^{6}$. Para llevar este tipo de concepción al campo de los entornos virtuales de aprendizaje, se ha tomado como paso primero (Erste Stufen) la reducción de los procesos de aprendizaje a la "resolución de problemas".

Quedan, entonces, las preguntas: ¿qué es un problema filosófico? ¿cómo se desarrollan habilidades cognitivas sobre él? ¿cómo se da la sucesión de "cambios de estado” desde el “estado inicial” hasta el "estado meta”?

Como ya se indicó, se ha adoptado la posición de L. Embree ${ }^{7}$ que muestra cómo el aprendizaje de la filosofía es, ante todo, correlativo al entrenamiento en lo que, sin más, puede ser llamado análisis reflexivo. Este, a su turno, es un peculiar desarrollo de lo que se ha caracterizado en la tradición filosófica como fenomenología. Ésta impone que no se trate de los problemas más que en cuanto conciernen al orden de la experiencia subjetiva de mundo.

Por otra parte, como Embree mismo lo implementa en su concepción, el desarrollo de la competencia analítica -reclamada por el análisis reflexivo- tiene que ver esencialmente con el uso del "pensamiento diagramático”: los problemas pueden ser reducidos a formas o diagramas que permiten su comprensión, bien bajo un sistema de diagramas de flujo, bien bajo otros esquemas o modelos de representación.

Al cabo, pues, partiendo de las consideraciones teóricas previas, cabe ver la confluencia entre "resolución de problemas", “fenomenología” -entendida como análisis reflexivo-, "pensamiento diagramático” y “representación de conocimiento”. La presente ponencia da no sólo cuenta de la implementación de los supuestos precedentes, sino que mira cómo se genera aprendizaje significativo en la filosofía.

\section{Entornos virtuales de aprendizaje como herramientas para el aprendizaje en el filosofar}

Se parte de la noción de "ambiente de aprendizaje”. Éste se define, entonces, como un conjunto de experiencias que permite que el estudiante de filosofía -para su formación profesional como investigador y como maestro-, de manera estructurada, ejerza procesos autónomos de construcción de conocimiento propios de la disciplina y, preferencialmente, habilidades de conocimiento dentro de ella.

Sin convertir la informática en ámbito exclusivo, esta investigación toma los “entornos virtuales" como unos ambientes en los que se da el ejercicio de aprendizaje autónomo de la filosofía. En consecuen-

4 Cf. VARGAS GUILLÉN, Germán. Filosofía, pedagogía, tecnología. Bogotá: Alejandría Libros, 2003, p. 236.

5 CASTRO, Harold. GÓMEZ, Rafael y RUEDA, Francisco. "Uso educativo de Internet: una aproximación pedagógica”. En: Revista informática educativa Uniandes Vol. 11, No. 2, 1998, pp. 14-24; SILVIO, José. La virtualización de la universidad. Caracas: IESALC-UNESCO, 2000.

6 EMBREE, Lester. Análisis reflexivo. Morelia: Jitanjáfora, 2003, pp. 11 y 33.

7 Ibíd. 
cia, las herramientas informáticas como entornos virtuales de aprendizaje son una alternativa adecuada para satisfacer los requerimientos de los estudiantes de la Universidad Pedagógica Nacional, pues ofrecen ventajas que potencian el aprendizaje con sentido analítico y crítico. Entre éstas se pueden mencionar:

- Favorecen el aprendizaje colaborativo, pues permiten la discusión de textos entre los estudiantes y entre estos y el profesor o grupo de profesores; permiten además la construcción de significados en grupo mediante herramientas propias de las plataformas on line (chat, carteleras de discusión, foros, teleconferencias, etc.) y de herramientas informáticas que permiten la implementación de estrategias didácticas como mapas conceptuales y redes semánticas.

- Permiten implementar estrategias didácticas propias de la filosofía-como la lectura comprensiva de textos, la redacción de ensayos, el comentario y la reseña, entre otras- que impliquen desafíos para los estudiantes; favorecen en éstos el desarrollo de su capacidad de análisis, de crítica y de interpretación, indispensables en el aprendizaje del filosofar. Todas estas estrategias, acaso desplegadas a partir de la metodología de "solución de problemas”, que también son propias de los entornos virtuales.

- Propician la presentación de información y conceptos haciendo uso de piezas hipermediales -como, por ejemplo, mapas conceptuales (con componentes gráficos que facilitan diversas formas de representación del conocimiento), videos, audio-que facilitan la compresión de los temas de estudio.

- Permiten mayor acceso del estudiante -con la disponibilidad de "bibliotecas" o "bibliografías virtuales", el cotejo de fuentes, la preparación de informes individuales a partir de aquél, etc.- a la ejercitación y al análisis individual de textos, independientemente del tiempo y del espacio destinados para la clase formal.

\section{Estrategias}

A partir de los requerimientos planteados anteriormente y de las ventajas que ofrece el uso de entornos virtuales de aprendizaje, se han desarrollado algunas estrategias para estudiantes de primero y segundo semestre de los programas curriculares de la Universidad Pedagógica Nacional. Tales estrategias involucran el uso de dispositivos como el libro de texto, lecturas complementarias, sistemas de representación del conocimiento (mapas conceptuales y redes semánticas) y herramientas de e-learning; todo esto bajo la orientación de un profesor (experto, con producción intelectual validada en el campo disciplinar objeto de la temática virtualizada) y el apoyo de profesionales tanto en la disciplina como en el uso de las tecnologías de la información aplicadas a la educación. A continuación se describen las características y la aplicación de estas estrategias:

- El libro de texto8: éste ofrece la visión del tema desde el punto de vista del experto. Se utiliza como una referencia básica para el desarrollo del proceso. Tiene, análogamente, el carácter de un manual para un curso ordinario. Se

8 Para esta fase del proyecto se han tomado las siguientes obras: VARGAS GUILLÉN, Germán. Pensar sobre nosotros mismos: introducción fenomenológica a la filosofía en América Latina. Bogotá: San Pablo, 2002; VARGAS GUILLÉN, Germán. Tratado de epistemología: fenomenología de la ciencia, la tecnología y la investigación social. Bogotá: San Pablo, 2003. 
mantiene, así, la referencia a una exposición sistemática de la materia, validada por la comunidad disciplinar -se sobreentiende, en este caso, de la filosofía; en concreto, la "introducción a la filosofía” como la “epistemología”.

- Las lecturas complementarias ${ }^{9}$ : presentan el mismo tema, visto por otros autores desde diferentes puntos que permitan al estudiante ir haciendo la construcción de su propio conocimiento. Se trata, en todos los casos, de “desdogmatizar” el punto de vista del manual, y consecuentemente el de su autor. Para tal efecto, la elección de esta lecturas se ordena, de manera explícita, a teorías rivales que permiten poner en cuestión las tesis del libro de texto.

- Los mapas conceptuales ${ }^{10}$ : se usan como una representación gráfica de conceptos, conectados significativamente por palabras que forman proposiciones de manera que dan sentido a un concepto específico.

La importancia del uso de mapas conceptuales en procesos de aprendizaje radica en el efecto de comunicación que produce entre estudiantes y profesor o experto en el tema; pues éstos los realizan los estudiantes. El profesor puede reconocer explícitamente lo que éstos han comprendido del tema. Los mapas construidos por expertos son objeto de cotejo con los elaborados por los estudiantes. De ahí se desprende la consabida "negociación de significados” que se orienta a lograr una mejor comprensión.

- Las redes semánticas: son estructuras de representación, no de conceptos y su distribución espacial jerárquicamente ordenada, sino de los significados que se dan en una teoría, que a su turno representa un conjunto de relaciones entre fenómenos (hechos, datos) del mundo.

Las redes semánticas son, pues, estructuras de la representación de la representación que los estudiantes se hacen del mundo, que expresan significados, relativizan teorías y comprensiones del mundo. Por tanto, más que instrumentos para la "negociación de significados”, son “dispositivos de conceptualización”.

- Herramientas e-learning ${ }^{11}$ : son dispositivos software que se encuentran disponibles en Internet y actúan como medios de comunicación sincrónica y asincrónica. Son especialmente útiles para comunidades de aprendizaje, pues a partir de estrategias didácticas previamente establecidas, favorecen aspectos como la cooperación en el proceso de aprendizaje, la conexión a información y datos disponibles en internet, y la comunicación bidireccional rápida, con independencia del tiempo y del espacio.

9 Éstas se han implementado en las modalidades tanto de "biblioteca virtual” como de "bibliografía virtual”. En todo caso, éstas tienen un carácter temático; procuran ofrecer información completa, actualizada, en castellano y en las lenguas originales, de los principales autores, textos y comentarios que apuntan al objetivo que se explica en las siguientes líneas.

10 Para este objetivo tiene particular importancia el programa cmap, disponible en la dirección electrónica http://cmap.coginst.uwf.edu. Por supuesto, se considera que ésta, como otras ayudas, son básicas para lo que en el contexto de esta investigación viene a ser la dimensión del "pensamiento diagramático" -con el que se aporta, como se dice más adelante, a la "poda de la verbosidad".

11 Para este fin se hace uso de la plataforma Manhattan Virtual Classroom que adquirió la U.P.N. para procesos de virtualización de sus programas académicos. Esta plataforma ofrece los siguientes servicios: chat, correo electrónico, foros de discusión, cartelera electrónica, enlaces a sitios web y publicación de calificaciones. 


\section{Aprendiendo a filosofar}

El proceso que siguen los estudiantes se orienta al desarrollo de las habilidades que se quieren potenciar en ellos. Para lograr este fin se ponen en práctica las estrategias mencionadas en el numeral anterior. Éste se describe a partir de las siguientes etapas:

1. A partir de una lectura inicial del libro de texto, el estudiante elabora, de manera individual, un mapa conceptual que representa su compresión del conocimiento. Este mapa es cotejado con el mapa construido por el experto para cada caso. Producto de la negociación de significados entre estudiantes y experto, el estudiante logra una comprensión inicial del tema y ejercita su capacidad de interpretación frente a las posturas de los otros expertos y estudiantes.

2. La segunda etapa consiste en abordar, de manera individual, lecturas complementarias que cuestionan la tesis de la lectura principal, y elaborar, en grupos de dos o tres estudiantes, mapas conceptuales que corresponden a estas lecturas. Esta etapa permite que los estudiantes ejerciten sus habilidades de interpretación y de argumentación.

3. Durante esta etapa se logra, de manera cooperativa, la construcción de redes semánticas que sirven como dispositivo de conceptualización del tema que se aborda. Esto da a los estudiantes las bases teóricas para emprender la siguiente etapa.

4. Por último, se plantean a los estudiantes situaciones problemáticas que puedan ser resueltas por ellos, de manera individual y grupal, haciendo uso de su comprensión del tema y de sus habilidades de análisis, interpretación y crítica.

\section{Validación}

El proceso que se ha llevado a cabo toma en consideración, en primer término, la coproducción entre "expertos”, “peritos” y “novatos". Se entiende por los primeros a los autores de libros de texto o manuales, que aportan una comprensión temática; por los segundos, los profesionales en la enseñanza de esos temas de la filosofía y los tecnólogos de la información para los procesos educativos; por los terceros, los estudiantes que acompañan el proceso de producción-validación.

Por tanto, los niveles de validación son:

En la comunidad de los filósofos: tomando para el caso de manera exclusiva manuales que se han reconocido en el mercado como objetos relevantes para la consecución de la meta de una enseñanza sistemática de la disciplina.

En la comunidad de los enseñantes de filosofía: tomando para el caso un grupo de licenciados en filosofía, con el debido entrenamiento previo tanto en la disciplina como en su enseñanza.

En la comunidad de los tecnólogos de la información aplicados a la educación: tomando para el caso profesionales de formación específica en la ingeniería de sistemas con aplicaciones al campo de la pedagogía.

En la comunidad de los aprendices: tomando para el caso los estudiantes-monitores que estatutariamente son vinculados a las investigaciones que se realizan profesionalmente en la Universidad Pedagógica Nacional. Concretamente, se trata de estudiantes vinculados a los proyectos "La automatización de la retórica II” (DCS00504) (de la línea de investigación Argumentación y mundo de la vida). 
En este sentido, el proyecto tiene realizada una fase de producción validada (1er. semestre de 2004) y anticipa una fase de experimentación pedagógica (2do. semestre de 2004). Ha empezado, pues, como un proyecto exclusivo de la Facultad de Humanidades, Departamento de Ciencias Sociales, pero se prevé su extensión, dentro del Proyecto de institucionalización del Departamento de Filosofía, a todos los programas curriculares de la Universidad pedagógica Nacional.

\section{Conclusiones}

- Resulta evidente que este proyecto, ante todo, es una “reconciliación” entre la tecnología y las humanidades. Se tiene, en su horizonte, la hipótesis de que los dispositivos tecnológicos -concretamente las tecnologías de "entornos virtuales para el aprendizaje”- propician una formación, más que en los contenidos de la disciplina, en las habilidades que permiten realizar el proyecto kantiano anunciado en el epígrafe.

- En segundo lugar, para un proyecto de formación pedagógica resulta de importancia que los maestros, en su proceso de apropiación tanto de habilidades como de conocimientos, hagan la experiencia de aprendizaje que se perfila como presupuesto del "mundo de la vida" que experimentan sus futuros estudiantes; esto es, en "entornos virtuales" -como tecnología vigente, con seguridad superada cuando estos formandos realicen su vida profesional.

- Filosóficamente, tanto los “entornos virtuales" como los procesos de discusión on line hacen que se experimenten de manera diferente categorías como las de subjetividad, intersubjetividad, cuerpo, otro, alteridad; y, al cabo, mundo de la vida.
- En contraposición, incluso, con una tradición secular de la filosofía, este proyecto -al hacer peculiar énfasis en la representación de conocimiento (diagramático, espacial, topológicamente, etc.) - tiende a “podar” la verbosidad y a lograr tanto precisión como síntesis en la elaboración de los conceptos.

- Finalmente, esta investigación -realizada con estudiantes del primer año de la carrera-tiende a ofrecer algoritmos para la enseñanza de la filosofía on line en la educación básica secundaria.

\section{Bibliografía}

ARISTÓTELES. Tratados de lógica. Estudio introductorio, preámbulos a los tratados y notas al texto de Francisco Larroyo. México: Porrúa, 1975.

AUSUBEL, David P. Psicología educativa. México: Trillas, 1976.

CASTRO, Harold, GÓMEZ, Rafael y RUEDA, Francisco. "Uso educativo de internet: una aproximación pedagógica”. En: Revista informática educativa Uniandes Vol. 11, No. 2, 1998, pp. 14-24.

EMBREE, Lester. Análisis reflexivo. Morelia: Jitanjáfora, 2003.

GLYMOUR, Clark. Thinking things throught: an introduction to philosophical issues and achievements. Cambrige: MIT Press, 1997.

GONZÁLEZ FLÓREZ, José y VARGAS GUILLÉN, Germán. "De la informática educativa a la pedagogía computacional: De J. Piaget \& S. Papert a A. Newell \& H.A. Simon”. En: Maestros pedagogos II: un diálogo con el presente. Medellín: Colegio Francés/ 
Corporación Región/ Fundación Confiar/ Corporación "Penca de Sábila", 1999, pp. 73-95.

KANT, Immanuel. Crítica de la razón pura (Trad., Pedro Ribas). Madrid: Alfaguara, 1989.

KHÖLER, Wolfgang. Psicología de la forma. Madrid: Biblioteca Nueva, 1972.

MALDONADO, Luis; GONZÁLEZ, José y VARGAS, germán. Ontologismo vs. logicismo. 1999. Inédito.

NEWELL, Allen y SIMON, Herbert A. "La ciencia de la computación”. En: Filosofía de la inteligencia artificial. México: F.C.E., 1994. vid: pp. 122-152.

NOVAK, J. D. y GOWIN, D. B. Learning how to learn. New York y Cambridge: Cambridge University Press, 1984.

PAPERT, Seymour. Desafío de la mente. Bs. As.: Eds. Galápago, 1981.

. La máquina de los niños. Barcelona: Paidós, 1993.

PIAGET, Jean. Estudios sobre lógica y psicología. Barcelona: Altaya, 1980.

RICH, Elaine y KNIGHT, Kevin. La inteligencia artificial. Madrid: Mc Graw Hill, 1996.

SILVIO, José. La virtualización de la universidad. Caracas: IESALC-UNESCO, 2000.

SKINNER, Burrus F. Tecnología de la enseñanza. Barcelona: Labor, 1985.
SOWA, J. "Semantics networks". En: Encyclopedia of artificial inteligence. Ed., Stuart C. Shapiro. New York: John Wiley \& Sons, 1987.

TURING, Alan M. "La maquinaria de la computación y la inteligencia”. En: Filosofía de la inteligencia artificial. Comp., M. Boden. México: F.C.E, 1994.

TURNER. Matemática moderna aplicada: probabilidades, estadística e investigación operativa. Capítulo 5: Teoría de grafos. Madrid: Alianza Universidad, 1974.

VARGAS GUILLÉN, Germán. Filosofía, pedagogía, tecnología. Bogotá: Alejandría Libros, 2003.

Pensar sobre nosotros mismos: introducción fenomenológica a la filosofía en América Latina. Bogotá: San Pablo, 2002.

La representación computacional de dilemas morales: investigación fenomenológica de epistemología experimental. Tesis doctoral dirigida por el prof. Luis Facundo Maldonado Granados. Bogotá: Universidad Pedagógica Nacional, 2004.

Tratado de epistemología: fenomenología de la ciencia, la tecnología y la investigación social. Bogotá: San Pablo, 2003.

VIGOTSKY, Lev S. Pensamiento y lenguaje. Barcelona: Eds. Fausto, 1998. 